\title{
Síndrome coronario agudo secundario a intoxicación por monóxido de carbono y respuesta al tratamiento con oxígeno hiperbárico
}

\author{
Jorge Guillermo Pérez Tuñón, ${ }^{1}$ Mayré Ivonne Bautista Albíter, ${ }^{2}$ Leslie Moranchel García, ${ }^{3}$ \\ Rocío Martiñón Ríos, ${ }^{4}$ Angélica Mila Hernández ${ }^{5}$
}

\section{Resumen}

El monóxido de carbono (CO) es un gas sin olor ni color que debe sus efectos asfícticos a su afinidad por proteínas transportadoras como la hemoglobina $(\mathrm{Hb})$. Se produce en todas las combustiones incompletas de materiales que contienen carbón. La intoxicación por CO representa una de las principales causas de daño neurológico y muerte alrededor del mundo ya que afecta primordialmente a los órganos con mayor demanda de oxígeno. El oxígeno hiperbárico $(\mathrm{OHB})$ es la terapia de elección en la intoxicación por $\mathrm{CO}$ y la única capaz de prevenir el desarrollo de secuelas en pacientes con datos de hipoxia. Presentamos el caso de una mujer con exposición accidental a $\mathrm{CO}$ que desarrolló un síndrome coronario agudo (SCA), la cual fue tratada exitosamente con $\mathrm{OHB}$.

Palabras clave: Intoxicación, monóxido de carbono, terapia con oxígeno hiperbárico.

\footnotetext{
1 Jefe del Centro Toxicológico Hospital Ángeles Lomas.

2 Toxicóloga Clínica adscrita al Servicio de Urgencias, Hospital Ángeles Puebla.

${ }^{3}$ Residente de segundo año del Postgrado de Alta Especialidad en Toxicología Clínica.

${ }^{4}$ Residente de primer año del Postgrado de Alta Especialidad en Toxicología Clínica.

${ }^{5}$ Residente de tercer año de Urgencias Médico-Quirúrgicas, Hospital General de Zona Núm. 8 IMSS.
}

Correspondencia:

Dr. Jorge Guillermo Pérez Tuñón

Correo electrónico: toxicología_angeles@yahoo.com.mx

Aceptado: 12-01-2017.

Este artículo puede ser consultado en versión completa en http:// www.medigraphic.com/actamedica

\section{Summary}

Carbon monoxide (CO) is an odorless, colorless gas that owes its asphyctic effects to its affinity for carrier proteins such as hemoglobin $(\mathrm{Hb})$. It is formed during the incomplete combustion of any carbon containing compound. CO poisoning is a major cause of neurological damage and death around the world, because it primarily affects the organs with the highest oxygen demand. Hyperbaric oxygen therapy $(\mathrm{HBO})$ is the first line treatment for $\mathrm{CO}$ poisoning and the only way to prevent development of sequelae in patients with hypoxia. We report a case of a woman with accidental exposure to $\mathrm{CO}$ who developed a coronary acute syndrome (CAS), which was successfully treated with $\mathrm{HBO}$.

Key words: Poisoning, carbon monoxide, hyperbaric oxygen therapy.

\section{INTRODUCCIÓN}

El monóxido de carbono (CO), es un gas sin olor ni color que se produce por la combustión incompleta de material orgánico o cuando no existe un adecuado aporte de oxígeno durante dicha combustión. Aunque es posible que se genere en el organismo por la degradación del grupo hemo, las principales fuentes de CO son externas. Dentro de las más comunes encontramos la quema de leña, el escape de los automóviles con ausencia o mal funcionamiento de sus carburadores, calentadores de agua, estufas, secadoras de gas, generadores de electricidad e incendios, entre otros. ${ }^{1,2}$

Una vez en el organismo, el CO representa una toxina celular que se une de forma reversible a la hemoglobina (Hb), debido a que la afinidad de ésta por el CO es 250 a 300 veces superior que por el oxígeno., 3 Dicha unión origina la formación de carboxihemoglobina $(\mathrm{COHb})$, una forma patológica de la hemoglobina que resulta incapaz de entregar oxígeno a nivel tisular causando hipoxia. A medi- 
da que se eleva la concentración de CO, este fenómeno se repite con otras globinas de importancia clínica, como sucede con la mioglobina, donde se muestra una afinidad 60 veces superior por el CO en comparación con el oxígeno, obteniéndose carboximioglobina, lo cual interfiere con la contractilidad miocárdica. Sin embargo, la afinidad por las globinas no es el único mecanismo por el cual el CO induce toxicidad: su afinidad por metaloproteínas que contienen hierro y cobre interfiere con la función de transportadores como citocromo C, una proteína esencial en la cadena respiratoria y cuya inhibición acentúa el estado de hipoxia. ${ }^{2-4}$ La hipoxia origina daño tisular y lesión producida por estrés oxidativo; este último se presenta de manera especial en el sistema nervioso central, razón por la que una gran parte de los pacientes intoxicados muestra alteraciones en los ganglios basales y la corteza cerebral. También contribuyen a perpetuar la isquemia, la acidosis metabólica (por elevación de lactato), la hipokalemia, la hipotensión arterial, la depresión respiratoria y el edema pulmonar. ${ }^{1} \mathrm{Al}$ ser el $\mathrm{CO}$ una molécula de señalización, actúa también de manera específica en SNC en el locus coeruleus y el núcleo del tracto del solitario, causando manifestaciones neurológicas; pero también, debido a que ambos sitios son primordiales para lograr el control autonómico del sistema cardiovascular, es que cantidades elevadas de CO causan una disminución de la tensión arterial y la frecuencia cardiaca. ${ }^{5}$

Los signos y síntomas dependen del grado de exposición y consisten en cefalea, mareo, náusea, vómito, astenia, adinamia, confusión, disnea, letargia, dolor abdominal, coma y crisis convulsivas. Con relación a las manifestaciones cardiovasculares, pueden presentarse angina, infarto, arritmias (cambios en el segmento ST, ondas T patológicas, taquicardia y fibrilación ventriculares, entre las más frecuentes), falla cardiaca con choque cardiogénico y asistolia. ${ }^{6}$

El tratamiento inicial consiste en la administración de oxígeno suplementario. El oxígeno normobárico es capaz de acortar la vida media de la $\mathrm{COHb}$ (de 4 horas a una media de 60 minutos) e incrementar la saturación de oxígeno. No obstante, ante la sospecha de isquemia $(\mathrm{COHb}$ $25 \%$ o mayor, alteraciones neurológicas, cefalea persistente, disfunción cardiovascular o acidosis metabólica), o bien en pacientes con alto riesgo de desarrollarla (menores de cuatro años o mayores de 65, embarazadas, con exposición prolongada o recurrente) si presentan una $\mathrm{COHb}$ mayor o igual a $15 \%$, el tratamiento de elección consiste en la administración de oxígeno hiperbárico, que además de reducir la vida media de la $\mathrm{COHb}$ (a 23 minutos en promedio), reduce la peroxidación lipídica, incrementa la captación de especies reactivas de oxígeno, reduce la cascada inflamatoria asociada al daño por isquemiareperfusión, favorece la reactivación de citocromo $\mathrm{C}$ a nivel mitocondrial e incrementa la disponibilidad tisular de oxígeno no dependiente de hemoglobina, siendo la única medida terapéutica efectiva para la prevención de la neuropatía tardía en la intoxicación por CO. Aunque la dosis óptima y la frecuencia del tratamiento con oxígeno hiperbárico es desconocida se propone un esquema de tres a cinco sesiones en un periodo de 72 horas a una presión interna de $3 \mathrm{~atm}^{7}$

Presentamos el caso de una paciente que desarrolló dolor precordial y alteraciones electrocardiográficas sugestivas de isquemia miocárdica luego de una exposición accidental a CO. ${ }^{1-4,7}$

\section{CASO CLÍNICO}

Paciente femenino de 54 años de edad, quien a su ingreso no contaba con antecedentes patológicos de importancia. Fue encontrada con pérdida del estado de alerta en el cuarto de lavado de su domicilio. Sus familiares refirieron perder contacto con ella durante aproximadamente 30 minutos, acudiendo en su búsqueda cuando la paciente no respondió a su llamado. Para su traslado al hospital solicitaron el apoyo de una ambulancia, los paramédicos le suministraron oxígeno suplementario en el trayecto al hospital durante aproximadamente 20 minutos.

Clínicamente se recibió estuporosa, con respuesta a estímulos dolorosos y adecuado esfuerzo ventilatorio; se reportaba una saturación de oxígeno al 100\% con oxígeno en máscara con reservorio $\left(\mathrm{FiO}_{2}: 40 \%\right)$. Durante el abordaje inicial, la paciente mostró una ligera tendencia a la recuperación del estado de alerta, siguiendo órdenes, respondiendo parcialmente al interrogatorio y refiriendo la presencia de dolor precordial opresivo de intensidad moderada, además de cefalea holocraneana pulsátil y náusea. Presentaba una herida cortante en labio inferior derecho, así como un hematoma subgaleal en región temporal derecha, sin que existiera otra alteración en la exploración física. La gasometría arterial inicial se reportó con acidosis metabólica descompensada con hiperlactatemia e hiperoxemia: $\mathrm{pH} 7.32, \mathrm{PaCO}_{2} 21.8 \mathrm{mmHg}, \mathrm{PaO}_{2}$ $105 \mathrm{mmHg}$, Lactato $10.3 \mathrm{mmol} / \mathrm{L}, \mathrm{BE}-13.9 \mathrm{mmol} / \mathrm{L}, \mathrm{HCO}_{3}$ $14.5 \mathrm{mmol} / \mathrm{L}, \mathrm{COHb} 47.9 \%$. El electrocardiograma con FC $108 \mathrm{lpm}$, eje a la izquierda, con un desnivel negativo de ST de V2 a V6, en DII, DIII y aVF (Figura 1). Fue solicitado un perfil de enzimas cardiacas, el cual se reportó dentro de parámetros normales (troponina I $<0.05 \mathrm{ng} / \mathrm{mL}$, CK-MB $<1.0 \mathrm{ng} / \mathrm{mL}$, mioglobina $100.0 \mathrm{ng} / \mathrm{mL}$, dímero D $371 \mathrm{ng} /$ $\mathrm{mL}$ y BNP $43.8 \mathrm{pg} / \mathrm{mL}$ ).

Con lo anterior se corrobora el diagnóstico de intoxicación por $\mathrm{CO}$ y síndrome coronario agudo, siendo candidata a tratamiento con oxígeno hiperbárico, por lo que la paciente es ingresada a una primera sesión de 90 minutos, 


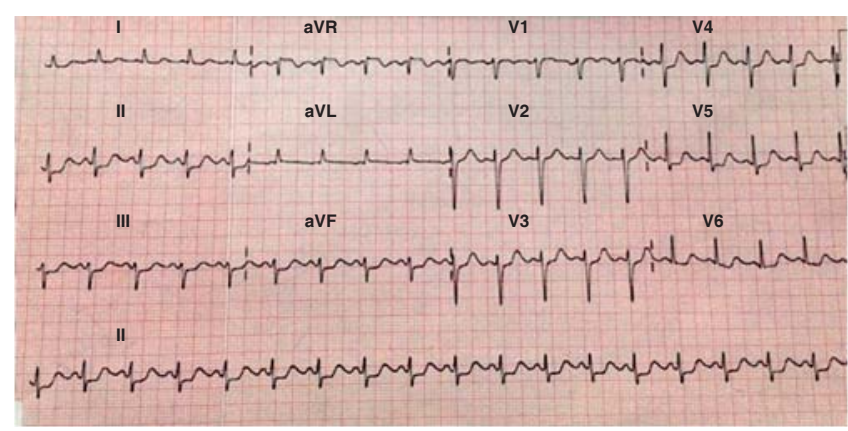

Figura 1. Electrocardiograma inicial donde se observa taquicardia y alteraciones del segmento ST de V2-V6, DII, DIII y aVF compatibles con isquemia.

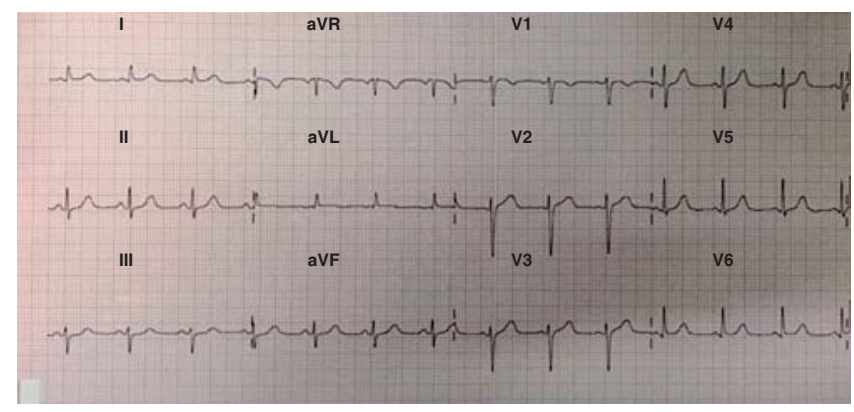

Figura 2. Electrocardiograma de control, posterior a una sesión en cámara hiperbárica de 90 min a 2.7 atm. Se observa normalización del segmento ST y la frecuencia cardiaca.

tolerando 2.7 atm de presión, aproximadamente a las dos horas de haber sido encontrada en su domicilio.

Una vez concluida la sesión se observa con mejoría clínica significativa, alerta, refiriendo remisión de la cefalea y la náusea, orientada en sus tres esferas. Se realiza exploración neurológica sin encontrar alteración en funciones mentales superiores, con memoria episódica, semántica y de trabajo conservada. Atención, lenguaje, cálculo, gnoxias y praxias, juicio y abstracción normales. Sin alteraciones en nervios craneales, fuerza y sensibilidad conservadas, sin dismetrías ni diadococinesias y marcha normal, sin reflejos patológicos ni movimientos anormales. Con minimental (MMSE) de 29/30 puntos (-1 punto en área de evocación). Se solicita control gasométrico, el cual se reporta con una incipiente alcalosis respiratoria compensada e hiperoxemia, con pH 7.44, $\mathrm{PaCO}_{2} 30.7 \mathrm{mmHg}, \mathrm{PaO}_{2} 297 \mathrm{mmHg}$, lactato $0.7 \mathrm{mmol} / \mathrm{L}, \mathrm{BE}-2.9 \mathrm{mmol} / \mathrm{L}, \mathrm{HCO}_{3} 22.7 \mathrm{mmol} / \mathrm{L}$, carboxihemoglobina 1.8\%. En esta ocasión se observó un electrocardiograma con FC $79 \mathrm{lpm}$, sin datos de isquemia, lesión o necrosis (Figura 2).

Se realizó además RM de cráneo con la finalidad de descartar cambios tempranos a nivel de ganglios basales, misma que se observó sin alteraciones, por lo que fue egresada a las seis horas desde la exposición para completar cinco sesiones de oxígeno hiperbárico en forma ambulatoria, así como revaloración por toxicología clínica.

\section{DISCUSIÓN}

La intoxicación por CO representa una de las principales causas de daño neurológico y muerte alrededor del mundo, siendo mayor su incidencia durante los meses fríos del año y dentro del domicilio de los pacientes. Debido a que no se detecta por los sentidos y a que los síntomas iniciales son inespecíficos es que existe un importante subregistro de esta patología. ${ }^{8}$ No obstante, sólo en Estados Unidos se reportan aproximadamente 50,000 casos de exposición al año, con alrededor de 5,000 letalidades, generando secuelas neurológicas en 30 a $70 \%$ de los pacientes expuestos. ${ }^{9}$

Si bien la mayor parte de las manifestaciones son neurológicas, la isquemia es un factor que puede afectar a cualquier tejido, especialmente aquellos que presentan una mayor demanda de oxígeno, como en el caso del miocardio. En este sentido, los pacientes que cuentan con factores de riesgo para desarrollar SCA (diabetes, obesidad, aterosclerosis, edad avanzada, etc.) son también quienes con mayor frecuencia presentan angina o infarto asociados a intoxicación por CO. ${ }^{6,10}$ En el caso de nuestra paciente, además del climaterio no se encontró otro antecedente relevante para el desarrollo del SCA, el cual logró identificarse mediante los hallazgos clínicos y electrocardiográficos.

Entre las complicaciones más importantes asociadas a isquemia se encuentra la necrosis de los músculos papilares que puede ser causa de insuficiencia cardiaca. ${ }^{11}$ Ello queda de manifiesto con la elevación de biomarcadores de necrosis miocárdica como CK-MB y troponina I. Dicha elevación comienza a ser evidente luego de cuatro a seis horas para CK-MB y de dos a cuatro horas para troponina I, desde el inicio de los síntomas. ${ }^{12,13}$ Debido a ello, y probablemente por la instauración temprana de la terapia con $\mathrm{OHB}$, en nuestra paciente dichos biomarcadores permanecieron inalterados.

El tratamiento consiste en retirar al paciente de la fuente de exposición, iniciar el aporte de oxígeno y si existe sospecha de isquemia iniciar la terapia con OHB lo más pronto posible. ${ }^{1-4}$ En el caso que se presenta, las directrices de tratamiento fueron llevadas a cabo con la paciente y pese a presentar un SCA ninguna otra medida contra la isquemia coronaria fue requerida en el periodo agudo.

Finalmente, se debe enfatizar que la intoxicación por $\mathrm{CO}$, pese al subregistro prevaleciente, es una condición frecuente, la cual se asocia al desarrollo de secuelas graves o la muerte y en la que el inicio oportuno del tratamiento puede representar un factor fundamental para el pronós- 
tico de los pacientes. Por lo anterior, el acceso ágil a un servicio de medicina hiperbárica debe ser considerado una prioridad por parte de los servicios de urgencias en los hospitales de segundo nivel.

\section{REFERENCIAS}

1. Hampson NB, Piantadosi CA, Weaver LK. Practice recommendations in the diagnosis, management, and prevention of carbon monoxide poisoning. Am J Respir Crit Care Med. 2012; 186 (118): 1095-1101.

2. Guzman JA. Carbon monoxide poisoning. Crit Care Clin. 2012; 28: 537-548.

3. Chiew AL, Buckley NA. Carbon monoxide poisoning in the 21st century. Crit Care. 2014; 18 (2): 221-228.

4. Prockop LD, Chichkova RI. Carbon monoxide intoxication: an updated review. J Neurol Sci. 2007; 262: 122-130.

5. Andresen MC, Doyle MW, Jin YH, Bailey TW. Cellular mechanisms of baroreceptor integration at the nucleus tractus solitarius. Ann NY Acad Sci. 2001; 940: 132-141.

6. Gandini C, Castoldi AF, Candura SM, Locatelli C, Butera R, Priori S et al. Carbon monoxide cardiotoxicity. J Toxicol Clin Toxicol. 2001; 39: 35-44.
7. Wolf SJ, Lavonas EJ, Sloan EP, Jagoda AS. Clinical policy: critical issues in the management of adult patients presenting to the emergency department with acute carbon monoxide poisoning. Ann Emerg Med. 2008; 51: 138-152.

8. Harper A, Croft-Baker J. Carbon monoxide poisoning: undetected by both patients and their doctors. Age and Ageing. 2004; 33: 105-109.

9. Iqbal S, Clower JH, Boehmer TK, Yip FY, Garbe P et al. Carbon monoxide-related hospitalizations in the U.S.: evaluation of a webbased query system for public health surveillance. Public Health Rep. 2010; 125: 423-432.

10. Jung Y, Lee J, Min Y, Yip FY, Garbe P. Carbon monoxide-induced cardiomyopathy. Epidemiology, clinical characteristics and prognosis. Circ J. 2014; 78: 1437-1444.

11. Aslan S, Erol MK, Karcioglu O, Meral M, Cakir Z, Katirci Y. The investigation of ischemic myocardial damage in patients with carbon monoxide poisoning. Anadolu Kardiyol Derg. 2005; 5: 189-193.

12. Satran D, Henry CR, Adkinson C, Nicholson CN, Bracha $Y$, Henry TD. Cardiovascular manifestations of moderate to severe carbon monoxide poisoning. J Am Coll Cardiol. 2005; 45: 1513-1516.

13. Kalay N, Ozdogru I, Cetinkaya Y, Eryol NK, Dogan A, Gul I et al. Cardiovascular effects of carbon monoxide poisoning. Am J Cardiol. 2007; 99: 322-324. 